\title{
Leiomyoma of the gallbladder in a patient with metastatic gastrointestinal stromal tumor in the liver: A case report with differential diagnostic considerations
}

\author{
MITSUAKI ISHIDA $^{1}$, HISANORI SHIOMI ${ }^{2}$, SHIGEYUKI NAKA ${ }^{2}$, TOHRU TANI ${ }^{2}$ and HIDETOSHI OKABE ${ }^{1}$ \\ ${ }^{1}$ Department of Clinical Laboratory Medicine and Division of Diagnostic Pathology; \\ ${ }^{2}$ Department of Surgery, Shiga University of Medical Science, Otsu, Shiga 520-2192, Japan
}

Received May 17, 2012; Accepted August 16, 2012

DOI: $10.3892 / \mathrm{ol} .2012 .910$

\begin{abstract}
Mesenchymal tumor of the gallbladder is rare and the majority of cases present as sarcomas. Benign mesenchymal tumors of the gallbladder are extremely rare and to date, only one case of leiomyoma has been reported. In this report, we describe a second case of leiomyoma of the gallbladder, and discuss the differential diagnostic considerations. A 55-year-old female with metastatic gastrointestinal stromal tumors (GISTs) in the liver was identified to possess a tumorous lesion of the gallbladder. The patient had previously received a partial gastrectomy for GIST, and multiple liver metastases were revealed. Following surgery, imatinib was administered, and the liver metastatic lesions were stabilized. Histopathological analysis of the resected gallbladder specimens revealed that the nodule arose from the muscular layer, and was composed of spindle cell proliferation with eosinophilic cytoplasm and bland cigar-shaped nuclei. Mitotic figures were rarely noted, and necrosis was not observed. Immunohistochemical analyses demonstrated that the tumor cells were positive for desmin and alpha-smooth muscle actin, but negative for CD117 and CD34. In accordance with these results, a final diagnosis of leiomyoma of the gallbladder was made. The present case is unique since the patient possessed multiple metastatic GISTs in the liver, and the gallbladder nodule was preoperatively suspected to be a metastatic GIST. Leiomyoma of the gallbladder is extremely rare; however, it may be an underrecognized entity. Thus, it is important to differentiate leiomyoma from GIST to avoid unnecessary clinical follow-up and treatment.
\end{abstract}

Correspondence to: Dr Mitsuaki Ishida, Department of Clinical Laboratory Medicine and Division of Diagnostic Pathology, Shiga University of Medical Science, Tsukinowa-cho, Seta, Otsu, Shiga 520-2192, Japan

E-mail: mitsuaki@belle.shiga-med.ac.jp

Key words: leiomyoma, gastrointestinal stromal tumor, gallbladder, liver metastasis

\section{Introduction}

Mesenchymal tumor of the gallbladder is rare, and the majority of cases present as sarcomas, which comprise approximately $2 \%$ of all malignant tumors of the gallbladder (1). Benign mesenchymal tumors of the gallbladder are extremely rare, but granular cell tumor is the most common type of benign mesenchymal tumor (2). Leiomyoma is one of the common mesenchymal tumors of the gastrointestinal tract, particularly in the esophagus and colon; however, only one case of leiomyoma of the gallbladder has been reported in the English-language literature (3). In this case report, we describe a second case of leiomyoma of the gallbladder in a patient with metastatic gastrointestinal stromal tumors (GISTs) in the liver and discuss the differential diagnostic considerations. The study was approved by the Ethics Committee of Shiga University of Medical Science., Shiga, Japan. Informed consent was obtained from the patient.

\section{Case report}

A 55-year-old female with a metastatic GIST in the liver was identified, using computed tomography (CT), to also possess a tumorous lesion of the gallbladder. The patient had received a partial gastrectomy for submucosal tumor of the stomach 4 years previously, and a CT scan revealed multiple metastatic lesions in the liver prior to surgery. The histopathological and immunohistochmical analyses of the resected stomach specimen identified a moderate risk GIST, according to the classification by Miettinen et al (4). The tumor size was $2.5 \mathrm{~cm}$, mitotic figures were $>5 / 50$ high-power fields, and immunohistochemical analysis revealed that the tumor cells were positive for CD117 and CD34, but negative for desmin and S-100 protein. Following surgery, imatinib was administered and subsequently, follow-up CT revealed that the number and size of the metastatic liver lesions were stable. A total of 4 years after the surgery, a tumorous lesion, measuring $8 \mathrm{~mm}$ in diameter, was identified in the neck of the gallbladder via follow-up CT. Metastatic GIST in the gallbladder was clinically suspected, thus, a laparoscopic cholecystectomy was conducted. The postoperative course was uneventful. 


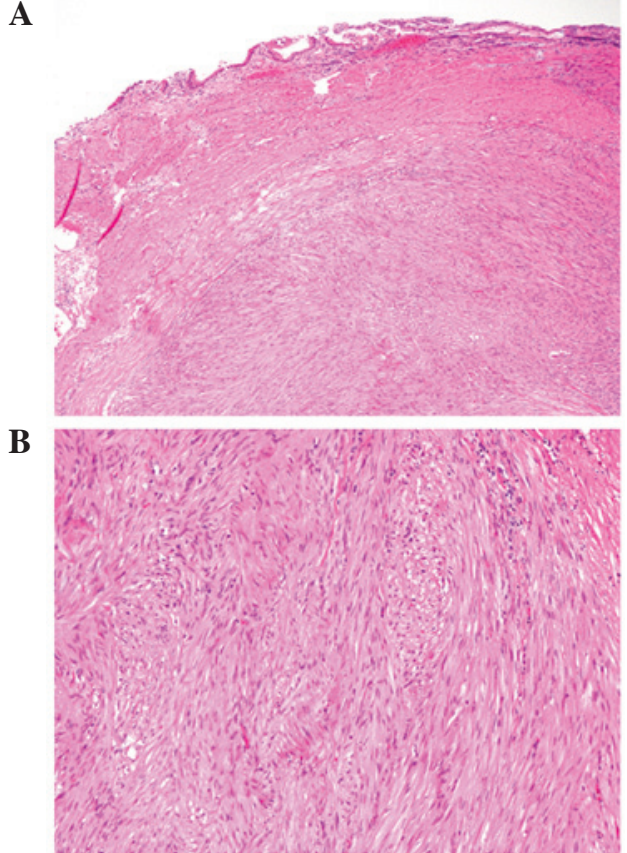

Figure 1. (A) A well-circumscribed proliferation of spindle cells in the gallbladder (hematoxylin and eosin staining; magnification, x40). (B) Spindle cells were arranged in intersecting fascicles with eosinophilic cytoplasm and bland cigar-shaped nuclei (hematoxylin and eosin staining; magnification, $\mathrm{x} 100)$.

Formalin-fixed, paraffin-embedded tissue blocks of the resected gallbladder specimens were cut into $3-\mu \mathrm{m}$ sections, deparaffinized and rehydrated. Each section was stained with hematoxylin and eosin, and then used for immunostaining and in situ hybridization. Immunohistochemical and in situ hybridization analyses were conducted using an autostainer (XT system Benchmark; Ventana Medical Systems, Tucson, AZ, USA) according to the manufacturer's instructions. The following primary antibodies were used: a mouse monoclonal antibody against alpha-smooth muscle actin (alphasm-1; Novocastra Laboratories, Ltd., Newcastle upon Tyne, UK), a mouse monoclonal antibody against CD34 (QBEnd/10; Novocastra Laboratories, Ltd.), a rabbit polyclonal antibody against CD117 (c-kit; DakoCytomation, Glostrup, Denmark), a mouse monoclonal antibody against desmin (D33; DakoCytomation), a mouse monoclonal antibody against h-caldesmon (h-CD; DakoCytomation), a mouse monoclonal antibody against Ki-67 (MM1; Novocastra Laboratories, Ltd.), and a rabbit polyclonal antibody against S-100 protein (Nichirei Bioscience, Tokyo, Japan). For in situ hybridization, an INFORM EBER (EBV-encoded early RNA) 1 probe (Ventana Medical Systems) was used.

Gross examination revealed a sharply demarcated intramural whitish nodule, measuring $8 \times 7 \mathrm{~mm}$ in diameter, in the neck of the gallbladder. Microscopically, the nodule was well-circumscribed and appeared to have arisen from the muscular layer of the gallbladder with pendulation into the mucosa. The nodule was composed of a proliferation of spindle cells arranged in intersecting fascicles, which contained eosinophilic cytoplasm and bland cigar-shaped nuclei with blunt edges (Fig. 1). Mitotic figures were rarely

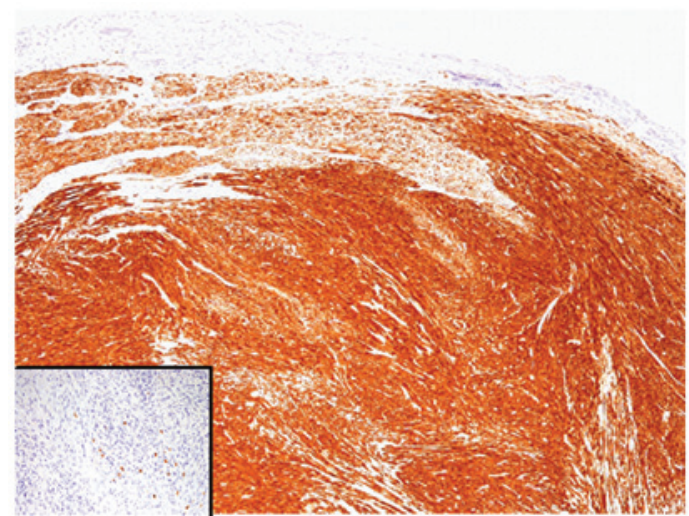

Figure 2. Immunohistochemical analysis revealed that spindle cells were diffusely and strongly positive for desmin (magnification, $\mathrm{x} 40$ ), but negative for CD117. Inset, CD117-positive mast cells are scattered within the tumor (magnification, $\mathrm{x} 100$ ).

noted ( $<1 / 50$ high-power fields), and necrosis was not observed. Immunohistochemical analyses revealed that tumor cells were strongly and diffusely positive for desmin, alpha-smooth muscle actin and $\mathrm{h}$-caldesmon, and negative for CD34, CD117 and S-100 protein (Fig. 2). The Ki-67 labeling index was $0.4 \%$. No EBER 1-positive cells were identified using in situ hybridization.

According to these histopathological and immunohistochemical findings, a final diagnosis of leiomyoma of the gallbladder was made.

\section{Discussion}

Here, we describe the second reported case of leiomyoma of the gallbladder in a patient with metastatic GIST in the liver. Wachter et al first reported a case of leiomyoma of the gallbladder in 2010 (3). The present case is unique since the patient had multiple metastatic GISTs in the liver, and the gallbladder nodule was preoperatively suspected to be metastatic GIST. Immunohistochemical results of the present case (CD117 negativity and desmin positivity) confirmed a diagnosis of leiomyoma.

Differential diagnosis of gallbladder leiomyoma includes leiomyosarcoma and GIST (3). Leiomyosarcoma is the second most common type of sarcoma in the gallbladder (6). In contrast to leiomyosarcoma, leiomyoma lacks nuclear atypia, pleomorphism, increased mitotic activity and necrosis, therefore, differentiation from leiomyosarcoma is not difficult. Epstein-Barr virus (EBV)-associated leiomyosarcoma/smooth muscle tumor occasionally involves the gallbladder in a setting of immunosuppression, either acquired or iatrogenic $(2,5)$. This type of tumor is characterized by the proliferation of oval to spindle-shaped mesenchymal cells, as well as positive and negative immunoreactivity for alpha-smooth muscle actin and desmin, respectively (5). EBER 1-positivity by in situ hybridization is also a characteristic feature (5). In addition, GIST of the gallbladder is rarely reported (6), and metastatic GIST should be also included in the differential diagnostic considerations, as in the present case. Although both leiomyoma and leiomyosarcoma may occasionally mimic GIST in morphology, the immunohistochemical characteristics of GIST can easily 
differentiate leiomyoma (CD117 negativity and desmin positivity) from GIST (CD117 positivity and desmin negativity) (5).

In conclusion, we report a case of leiomyoma of the gallbladder. Leiomyoma of the gallbladder is extremely rare; however, it may be an underrecognized entity. It is important to differentiate leiomyoma from GIST to avoid unnecessary long-term clinical follow-up and treatment.

\section{References}

1. Duffy A, Capanu M, Abou-Alfa GK, et al: Gallbladder cancer (GBC): 10-year experience at Memorial Sloan-Kettering Cancer Centre (MSKCC). J Surg Oncol 98: 485-489, 2008.

2. Miettinen M, Fletcher CDM and Kindblom LG: Mesenchymal tumours of the gallbladder and extrahepatic bile ducts. In: WHO Classification of Tumours of the Digestive System. Bosman FT, Carneiro F, Hruban RH and Theise ND (eds). IARC, Lyon, pp277, 2010 .
3. Wachter DL, Büttner MJ, Grimm K, Hartmann A and Agaimy A: Leiomyoma of the gallbladder: a case report with review of the literature and discussion of the differential diagnosis. J Clin Pathol 63: 177-179, 2010.

4. Miettinen M and Lasota J: Gastrointestinal stromal tumors: pathology and prognosis at different sites. Semin Diagn Pathol 23: 70-83, 2006.

5. Al-Daraji WI, Makhlouf HR, Miettinen M, et al: Primary gallbladder sarcoma: a clinicopathologic study of 15 cases, heterogeneous sarcomas with poor outcome, except pediatric botryoid rhabdomyosarcoma. Am J Surg Pathol 33: 826-834, 2009.

6. Peerlinck ID, Irvin TT, Sarsfield PT and Harington JM: GIST (gastro-intestinal stromal tumour) of the gallbladder: a case report. Acta Chir Belg 104: 107-109, 2004. 